\title{
Le voyage des compagnons : l'exemple des tours de France de Jacques-Louis Ménétra, compagnon vitrier au XVIIIe siècle
}

Companions and their journeys: the example of the tour of France of JacquesLouis Ménétra, a companion glazier in the 18th century

\section{Frédérique Pitou}

\section{(C) OpenEdition \\ Journals}

Édition électronique

URL : http://journals.openedition.org/abpo/2838

DOI : $10.4000 / a b p o .2838$

ISBN : 978-2-7535-3601-2

ISSN : 2108-6443

Éditeur

Presses universitaires de Rennes

Édition imprimée

Date de publication : 15 novembre 2014

Pagination : 43-58

ISBN : 978-2-7535-3450-6

ISSN : 0399-0826

Référence électronique

Frédérique Pitou, «Le voyage des compagnons : l'exemple des tours de France de Jacques-Louis Ménétra, compagnon vitrier au XVIIle siècle », Annales de Bretagne et des Pays de l'Ouest [En ligne], 121-3 | 2014, mis en ligne le 15 novembre 2016, consulté le 19 avril 2019. URL : http:// journals.openedition.org/abpo/2838; DOI : 10.4000/abpo.2838 


\title{
Le voyage des compagnons : l'exemple des tours de France de Jacques-Louis Ménétra, compagnon vitrier au XVIII siècle
}

\author{
Frédérique PITOU \\ Professeur d'Histoire moderne, université du Maine - CERHIO UMR 6258
}

Le monde du compagnonnage présente quantité de déplacements de ville en ville, de parcours individuels que l'historien saisit difficilement, sinon dans les fonds de justice, car les " affaires des compagnons " sont le plus souvent interdites, donc condamnées. Les archives de justice de la Mayenne présentent ainsi, pour la ville de Laval, au XVIII ${ }^{e}$ siècle, quelques affaires où il est question de cabales, de taxes, de débauches (dépenses au cabaret), de boutiques interdites ${ }^{1}$. Un billet, de 1769 , interdit une boutique en ces termes :

" Baraque defandue par troj jollis garsons et fait defance à qui que foit de bons garson du tour de France d'antre che sa flame marechalle de Lavalle et gargotte a la soupe aux lar et inquapable de pouvoir avoir auqun jolli garson marechaux et fait par nous le deux renoir et Louis tous bons garsons maréchaux à Laval ${ }^{2}$."

Il est également question d'assemblées de compagnons et de devoir, de violences (les "fricassées "), de certificats ou reconnaissances de bonne conduite qui doivent permettre de poursuivre un parcours :

« De Laval, le 10 mars 1727 (écrit 17027) Mes frères, nous vous faisons asavoir que il s'est arivé un compagnon nommé sant chagrain Langevain dont nous l'avons reconu pour honêste conpangon nous vous prions de le leser paser sans lui faire tor non plus qua vous même

" (siné brin d'amour le manceau, brin d'amour le manceau, sant chagrain le nantes, la jeunesse le normand, la traverse le manseau, la jeunesse le nor-

1. PIтоu, Frédérique, Laval au XVIII siècle. Marchands, artisans, ouvriers dans une ville textile, Laval, Société d'Archéologie et d'Histoire de la Mayenne, 1995, p. 482 sqq.

2. Arch. dép. de la Mayenne, B 946. 
mand, grosbois le normand, la jeunesse le manceau, la forge le manceau, la fleur le manceau $)^{3}$."

On a le sentiment là, pour ces compagnons, d'une vie parallèle sur laquelle on n'a d'informations qu'occasionnellement et en creux, lorsque la justice se saisit de leurs actions, bien qu'elle n'ait, selon les compagnons eux-mêmes, "rien à voir dans les devoirs des compagnons " ${ }^{4}$. D'où l'intérêt du témoignage de Ménétra ${ }^{5}$, d'autant plus précieux qu'il évoque le $\mathrm{XVIII}^{\mathrm{e}}$ siècle, alors que les témoins de Barret et Gurgand dans Ils travaillaient la France $^{6}$ sont des compagnons du XIX siècle (parmi eux le menuisier Agricol Perdiguier ${ }^{7}$ ). Le titre qui figure sur la première page du récit de Ménétra est : Journal de ma vie, écris pour moi en l'an 1764. Il affirme donc avoir commencé à écrire lors de cette année 1764 et il aurait achevé son journal en 1803. Les différentes phases de sa vie sont une enfance parisienne (il est né à Paris en 1738, il est fils d'un maître vitrier), une jeunesse turbulente comme compagnon, puis une vie d'artisan vitrier (il rentre définitivement à Paris en 1764 et se marie en 1765). La phase qui m'intéresse ici est donc celle de ses voyages (il quitte Paris le 29 mars 1757, revient rapidement puis repart pour faire son tour de France).

Le titre de ce colloque étant " Heurs et malheurs des voyages. Les conditions de voyage aux XVI ${ }^{\mathrm{e}}$-XVIII ${ }^{\mathrm{e}}$ siècles ", je me suis demandé quoi retenir, pour y répondre, du témoignage de Ménétra, car l'imaginaire commun associe l'expression de Tour de France à celui de voyage. C'est bien la pratique du déplacement, du voyage, qui confère à l'institution compagnonnique l'essentiel de sa notoriété. Le Tour de France est une institution sociale, c'est une instance de formation et d'initiation, mais c'est aussi en soi un voyage ${ }^{8}$. Autrement dit, ce qui arrive dans les villes du Tour fait évidemment partie du voyage. Ménétra parle en effet de ses différents séjours, beaucoup de ses distractions (les filles, les débauches, les blagues), un peu de son travail (dans les églises, les couvents, les châteaux). J'essaierai cependant de me consacrer en priorité au voyage en tant que déplacement de ville en ville. Je verrai donc successivement que le Tour de France est une succession

3. Arch. dép. de la Mayenne, B 1013.

4. Arch. dép. de la Mayenne, B 1013.

5. Journal de ma vie. Jacques-Louis Ménétra, Compagnon vitrier au $18^{e}$ siècle, présenté par Daniel Roche, Paris, Montalba, 1982, 431 p. Le récit de Ménétra est aujourd'hui inséparable des commentaires de Daniel Roche. Ses voyages occupent les pages 48 à 137, soit 89 pages sur 285.

6. BARret, Pierre et GuRgand, Jean-Noël, Ils voyageaient la France. Vie et traditions des compagnons du Tour de France au XIX $X^{e}$ siècle, Hachette, Livre de poche, 1980, 467 p.

7. Lui-même auteur des Mémoires d'un compagnon, présentées par Maurice AGUlHON, éditions de l'Imprimerie Nationale, 1992.

8. Dans un chapitre intitulé "Le Tour de France ", Étienne Martin Saint-Léon écrit : "Pendant des siècles et jusqu'à une époque récente, la principale, sinon l'unique raison d'être du compagnonnage a résidé dans la nécessité d'assurer au travailleur une direction et un appui pendant les années où il voyageait pour parfaire son éducation professionnelle ", Étienne MARTIN SAInT-LÉOn, Le compagnonnage. Son histoire, ses coutumes, ses règlements et ses rites, Jean-Cyrille Godefroy éd., 2010, 371p. (1 ${ }^{\text {re }}$ édition 1901). 
d'étapes, puis les conditions matérielles des déplacements, enfin « les heurs et malheurs " qui arrivent pendant ses voyages.

\section{Le Tour de France est une succession d'étapes ${ }^{9}$}

On peut rappeler les étapes du voyage de Ménétra (en italique les lieux où il séjourne et travaille, alors qu'il passe simplement par certaines autres villes) : Orléans, Paris, Versailles, Orléans, Vendôme et environs, Tours, Angers, Niort, Poitiers, Saint-Malo (se rend à l'île d'Yeu en bateau), Niort, Montreuil-Bellay, Nantes, La Rochelle, Rochefort (prend la route de Brest), Royan, Bordeaux, Agen, Auch, Toulouse et environs, Bayonne, Bordeaux, Agen, l'Isle Jourdain (Toulouse), Narbonne, Perpignan, Béziers, Montpellier, Nîmes, Marseille et environs, Carpentras, Avignon, Bédoin, Avignon, PontSaint-Esprit, Le pont du Gard, Montélimar, Valence-Crest, Lyon, Dijon, Auxerre.

Il suit ainsi les grandes routes ${ }^{10}$, c'est d'ailleurs un terme qu'il emploie fréquemment, dans le sens inverse des aiguilles d'une montre. Au siècle suivant, (il voyage en 1824-1828), Agricol Perdiguier (Avignonnais la Vertu) fait quasiment le même voyage, mais dans l'autre sens. La durée de ses séjours est variable, il passe plusieurs fois quinze jours au même endroit mais parfois beaucoup plus longtemps : lors de son second voyage, il reste presque une année à Lyon (onze mois). Chacune de ces étapes suppose un départ et une arrivée.

\section{Départ}

Le texte de Ménétra livre un certain nombre de récits de départ, largement semblables et pour lesquels les mêmes mots sont utilisés. Partir, pour le compagnon, c'est " se mettre en route " ou " prendre la route ". Ce départ est parfois annoncé et connu de l'entourage, parfois plus au moins précipité, comme lorsqu'il quitte Poitiers, où il a été, avec d'autres compagnons, injustement accusé d'avoir dégradé de jeunes arbres sur la voie publique et pour cela jeté en prison. Lorsqu'il est libéré, il ne s'attarde pas : " je fus chez la mère chercher mon sac et sans dire adieu à personne je me mis en route" (p. 59).

9. « Je partis pour faire mon Tour de France ", Journal de ma vie. Jacques-Louis Ménétra, Compagnon vitrier au $18^{e}$ siècle, présenté par Daniel Roche, Paris, Montalba, 1982, p. 46. Tous les emprunts au texte de Ménétra qui figurent dans cet article, et qui sont référencés entre parenthèses, sont extraits de cette édition. Dans un avertissement, Daniel Roche précise les principes qu'il a adoptés lors de l'édition, parmi lesquels le respect de l'orthographe parfois phonétique et de l'absence de ponctuation du manuscrit.

10. On voit bien que le compagnon emprunte les grands axes lorsque l'on compare son itinéraire avec la carte "Les grandes voies de communication, 1789 ", p. 15 de l'Atlas de la Révolution, fascicule 1 : Routes et communication, sous la direction de Guy ARBELLOT et Bernard LEPETIT, Paris, Éditions de l'EhEss, 1987. 
Le premier voyage de Ménétra (1757-1763)

(Source : Journal de ma vie. Jacques-Louis Ménétra, Compagnon vitrier au $18^{e}$ siècle, présenté par Daniel Roche..., op. cit., p. 55)

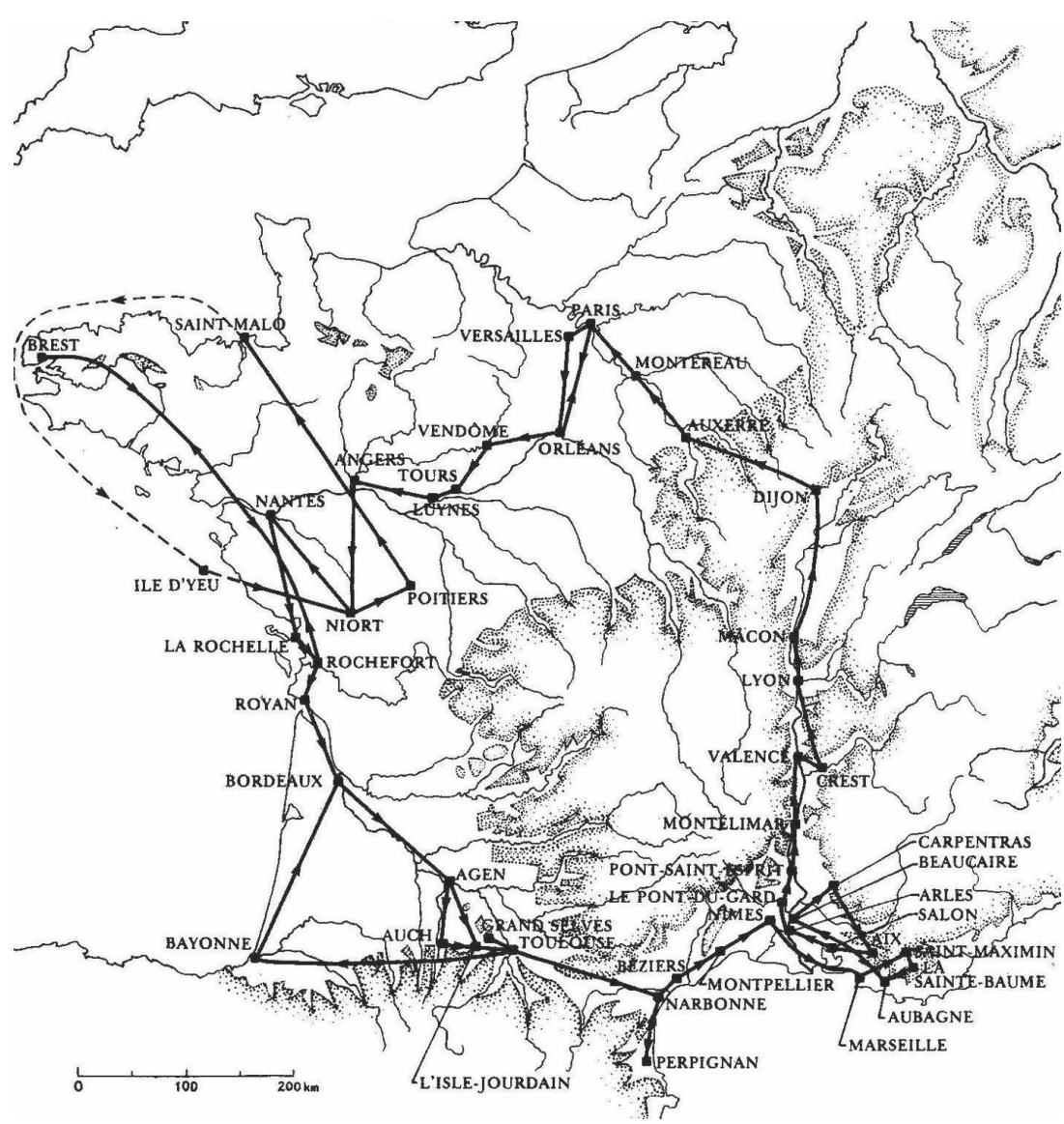

Ménétra se souvient de son premier départ, il ne ressent pas l'allégresse d'un Toussaint Guillaumou ${ }^{11}$ mais au contraire un sentiment de crainte qu'il exprime en des termes assez convenus : " je regardais souvent en arrière et il me semblait que les passants disaient que j'avais peur d'abandonner le clocher de mon village " (p. 41). Il quitte Paris mais il tient à cette expression de « village " car il la reprend lors de son second départ où il se

11. «Par une belle matinée du mois de mai 1830 [...] un petit bonhomme sortait pédestrement de la ville de Toulouse [...]. Les passants qui rencontraient ce voyageur en herbe se disaient sans doute, en le voyant si alerte et si guilleret : Voilà certes un mortel bien heureux ", Gulllaumou, Toussaint, Les confessions d'un Compagnon, dit Carcassonne Le Bien Aimé du Tour de France, Paris, Jacques Grancher éditeur, 1996, p. 23. 
montre plus vaillant : " je n'avais plus crainte de perdre le clocher de mon village " (p. 46).

Les causes du départ sont multiples; au premier rang, la volonté de se former, de voyager à travers les diverses provinces pour parfaire son éducation professionnelle ${ }^{12}$. Lorsque le compagnon est lancé dans son Tour, le départ d'une ville pour une autre se fait sans autre raison que le temps qui passe. Il ne séjourne pas trop longtemps dans une même ville car faire son Tour de France, c'est apprendre de nouvelles pratiques, donc voyager, quoiqu'il lui en coûte parfois, par exemple il écrit à propos de son départ de Tours pour Angers : " je regrettai cette charmante ville " (p. 53); ou à propos de son départ de Niort : " je ne pouvais point rester si longtemps dans la même ville " (p. 62).

Les étapes du Tour de France sont logiquement liées au travail et à la situation de l'emploi. Il se rend à Versailles car " il y avait un maître qui y avait besoin d'un compagnon" (p. 46). Dans cette ville, il fait la rencontre d'un compagnon qu'il connaît et qui travaille chez le vitrier du roi. Il reste trois mois dans ce travail, puis se rend à nouveau à Orléans, va voir " son ancien bourgeois ", sans doute le maître chez lequel il avait travaillé lors de son précédent séjour à Orléans, lequel l'envoie à Vendôme, "chez une bonne veuve dont le mari était défunt depuis six semaines" (p. 46).

En raison peut-être de sa réputation (c'est ce que Ménétra lui-même nous incite à penser), le compagnon est parfois appelé par un maître. Ainsi, lorsqu'il se rend à Nantes venant de Montreuil-Bellay, il est attendu : " je reçus une lettre de Nantes qu'un Guépin me faisait savoir que si je désirais demeurer chez une veuve qu'il lui avait dit beaucoup de bien " (p. 62). De la même façon, il se rend à Montpellier après avoir reçu une proposition précise : " étant à Béziers arriva une lettre qui m'était adressée pour venir à Montpellier de la part d'un maître chaudronnier qui avait entrepris les lanternes de Montpellier au nombre de six cents" (p. 83). La demande ne lui est parfois pas adressée personnellement, mais il apprend simplement que dans une autre ville, il y a du travail; ainsi, il séjourne à Lyon depuis onze mois lorsqu'il apprend qu'" un maître vitrier de Macon a écrit à la mère de Lyon pour lui demander trois compagnons " (p. 129). Malgré le projet de son bourgeois de l'établir à Lyon, il quitte la ville, son maître et toute sa famille, ses compagnons lors d'adieux qu'il nous décrit comme déchirants ${ }^{13}$.

Le départ d'une ville signifie la fin d'une période de travail, il suppose un accord avec le patron. Le compagnon et son maître " font les comptes";

12. «En partant sur le Tour de France, le jeune compagnon verra du pays et se formera: il aura l'occasion de mettre en œuvre son savoir, de le perfectionner et de le compléter ", PoITRINEAU, Abel, Ils travaillaient la France, Métiers et Mentalités du XVI au XIXe siècle, Armand Colin, 1992, p. 65.

13. Il écrit de son bourgeois " les larmes lui sortaient des yeux ", et se décrit lui-même ému : " Je les quittai en pleurant. » Chez Ménétra, la peine liée à l'arrachement du départ est fréquemment évoquée en termes forts mais semble vite dissipée. 
lorsqu'ils ne tombent pas d'accord, et cela arrive quelquefois, les consuls sont saisis, ainsi à Carpentras, Ménétra et ses compagnons font comparaître en justice un maître qui refuse de les laisser partir, ce qui permet au Parisien d'asséner cette vérité qui est visiblement plus pour lui qu'une formule toute faite, que "dans la France l'on était libre " (p. 93). Si le maître refuse de les laisser partir, c'est que l'ouvrage qu'ils ont commencé n'est pas achevé, mais les compagnons disent avoir été embauchés au mois et le tribunal leur a donné raison. Ces recours aux tribunaux ne sont pas fréquents, mais il y a des règles que les deux parties doivent respecter. Ainsi, il est défendu aux maîtres d'embaucher un compagnon sans s'assurer qu'il est en règle avec son ancien maître (ou le compagnon doit " battre la campagne ", c'est-à-dire rester un certain temps sans travailler). Cette défense de " débaucher " un compagnon laisse imaginer une situation qui n'est pas très favorable aux maîtres.

Mais parfois, pour le compagnon, partir n'est pas très différent de fuir. Le premier départ de Ménétra s'explique en grande partie car il ne s'entend pas avec un père violent (p. 40). D'une ville où il séjourne, il décampe parfois devant les conséquences de ses actes avec les filles. Il décrit ainsi les raisons de son départ pour Tours : « la sœur de la veuve me pressait de tenir la promesse que j'avais (faite) partie pour rire et partie pour avoir Comme j'ai assez promis et que je n'y tenais guère je m'en fus à Tours " (p. 49). Avant son départ d'Angers, Ménétra écrit : " il me tardait de quitter cette ville mes deux bonnes amies commençaient à s'apercevoir que le jupon faisait la bosse plus qu'à l'ordinaire " (p. 56). Ménétra se présente comme l'irresponsable parfait.

Il arrive également que Ménétra soit obligé de partir pour fuir des ennuis judiciaires, à la suite des bagarres auxquelles il est mêlé. C'est encore à Angers que Ménétra situe le récit extraordinaire d'une bataille épique entre compagnons : «il fut décidé un jour pour une batterie générale qui fut commandée pour le jour de la Saint-Barthélemy "; la conséquence est que "les chefs nous fûmes décrétés de prise de corps " et que « les gardes de la maréchaussée étaient après nous " (p. 56 et suivantes) ${ }^{14}$. À Nantes, il est pris dans une rixe, il est recherché par la police et sa bourgeoise (sa " bonne veuve ") est obligée de le cacher; il préfère donc partir (p. 65). À Lyon, il est recherché pour s'être battu avec un ancien compagnon devenu soldat et prend le parti de s'en aller (p. 120).

On trouve bien, dans le récit de Ménétra, des références à des villes agréables, qu'il quitte avec regret mais on sent bien que sa tendance naturelle est de voyager. Il invoque la volonté de voir des amis, comme lorsqu'il quitte Niort après un séjour de trois mois : " je voulus aller à Poitiers voir de mes amis " (p. 58). Lorsqu'il pense à rentrer, c'est pour retrouver sa

14. Si le déroulement de la bataille est assez bien décrit par Ménétra, les causes semblent être l'escalade à partir d'un incident qui a opposé des compagnons d'associations différentes, à tel point, écrit-il, que "lorsqu'on se rencontrait c'était toujours à se chamailler". 
famille, il dit ainsi à un aubergiste, du côté d'Arles : " que j'allais voir ma famille et qu'il y avait environ sept ans qu'ils ne m'avaient vu " (p. 100). Il profite de ses déplacements pour rendre visite à des personnages qu'il ne voit pas souvent, il va retrouver une tante à Valence (p. 102). L'objet de son second voyage est de retrouver sa "veuve de Nîmes ", auprès de laquelle il a l'intention de s'établir, après l'avoir épousée (p. 116).

Il fait parfois étape simplement pour le plaisir, il écrit ainsi de l'un de ses séjours : "J'avais un compagnon qui y était établi et je restais quelques temps à me délasser. "Quelques échappées témoignent de ce que Daniel Roche appelle une " attraction touristique ${ }^{15}$ ". Lorsqu'il est à Toulouse, il envisage de passer en Espagne : "Comme j'étais en argent [...] il me prit envie d'aller à Saint-Jacques. " Ce voyage est abandonné car il fait la rencontre de voyageurs qui en reviennent : "Nous vîmes arriver des compagnons qui en revenaient et qui étaient dans un état minable " (p. 78).

Entre deux villes ou autour d'une ville, il cherche à en visiter les principaux monuments et les alentours, à Marseille, il va voir la sainte Baume ${ }^{16}$ et la foire de Beaucaire; il fait l'ascension du Mont Ventoux à partir de Bédoin; il s'arrête à Salon pour voir le tombeau de Nostradamus; entre Pont-Saint-Esprit et Montélimar, il va voir le pont du Gard; entre Lyon et Macon, il veut aller voir Genève... Le but est toujours de juger si ce que l'on raconte traditionnellement de ces lieux est conforme à ce qu'il ressent et se dessine ainsi une série de vignettes touristiques qui composent la mémoire du compagnon et qu'il saura associer, dans ses récits futurs, à certaines de ses expériences personnelles.

La direction que suit Ménétra est parfois infléchie par des rumeurs qui circulent sur des affaires rentables, sur des gains faciles. Il entend par exemple parler de situations qui rapportent et va à Saint-Malo pour s'embarquer : "Nous avions entendu parler qu'il y avait de nos camarades qui dans trois mois avaient eu jusqu'à 800 francs " (p. 59).

Dans un long voyage, c'est parfois le hasard qui décide : "Comme je prenais le chemin et que j'hésitais si je n'irais pas à Toulouse ou à Bordeaux parce que la grande route fait la fourche à un postillon qui me demanda où j'allais je lui dis que je ne savais pas quel parti prendre il me dit qu'il allait à Bordeaux. " Ménétra choisit là de monter à l'arrière de sa voiture (p. 78-79).

Les départs sont l'occasion d'un rite auquel, visiblement on n'échappe pas, Ménétra rapporte un nombre important de " conduites ", ainsi lorsqu'il quitte Tours : "les compagnons [me] firent la conduite avec des violons et de hautbois" (p. 52); à Nantes, c'est une soixantaine de compagnons qui l'escortent à la sortie de la ville (p. 65); au départ de Carpentras, "ce

15. Roche, Daniel, Jacques-Louis Ménétra, Une manière de vivre au XVIII siècle, commentaire placé à la suite du Journal de Ménétra, p. 365.

16. La sainte Baume, dans la tradition compagnonnique, est le lieu où maître Jacques a été assassiné. Pour la tradition chrétienne, c'est un pèlerinage sur le lieu où s'est retirée Marie-Madeleine. 
fut par un dimanche que je quittai cette ville charmante accompagné de presque tous les compagnons de la ville de toutes les vacations ayant chacun le verre et la bouteille à la main nous fîmes le devoir " (p. 93). Dans ces nombreux récits de conduites, on trouve le plus souvent les éléments suivants : les compagnons qui restent dans la ville saluent celui qui poursuit son voyage, c'est l'occasion pour tous de jouer ou d'écouter du violon et de boire un verre. Il arrive que Ménétra emploie le mot pour qualifier un acte individuel; lorsqu'il quitte Auch, en parlant de son maître, il écrit : « il me fit la conduite avec la plus grande amitié et je repris la grande route pour aller à Toulouse " (p. 74). Il emploie aussi ce mot pour qualifier l'adieu de tout un village : lorsqu'il quitte Aucamville (près de Toulouse) : " Les bonnes gens d'Aucamville me firent la conduite en pleurant et le tambour battant" (p. 77).

\section{Arrivée}

Pour le compagnon voyageur qui arrive dans une ville, l'embauche est en général rapide, le placement des ouvriers étant la principale raison de l'existence de cette organisation du Tour de France. Lors de son arrivée à Tours, Ménétra écrit : " Je descendis chez la mère des compagnons qui me reçurent très bien et me mirent dans une bonne boutique de la ville " (p. 50). Plus précisément encore, il écrit de son arrivée à Poitiers : "J'arrivais un dimanche matin et fus embauché pour travailler le lendemain " (p. 59).

Les compagnons sont en effet pris en charge par un réseau que l'on repère dans le texte de Ménétra; celui-ci évoque dans toutes les villes où il s'arrête les auberges compagnonniques tenues par la mère, ou le père, qui accueillent les compagnons qui circulent. Ces aubergistes sont initiés au devoir, ils représentent l'autorité compagnonnique et lui sont liés par un contrat. Cette auberge est en quelque sorte un foyer pour les jeunes ouvriers, où ils trouvent gîte, couvert, et solidarité. Aussi sommaire qu'elle soit, elle comporte un réfectoire, un dortoir et un lieu de réunion nommé " cayenne ", c'est ce que disent les historiens du compagnonnage ${ }^{17}$, mais Ménétra n'utilise jamais le terme. De même qu'il ne parle pas d'un personnage auquel est dévolu un rôle important puisqu'il fait connaître les nouveaux arrivants aux maîtres qui offrent du travail : le rouleur. Lorsqu'il tient lui même ce rôle, à Lyon notamment, il se qualifie de " premier en ville ". En revanche, il évoque souvent autour de ces auberges où sont réunis les compagnons, des valeurs de solidarité, d'entraide ou d'assistance. Ménétra est compagnon du devoir. Les compagnons voyageurs appartiennent en fait à trois compagnonnages ou "devoirs " concurrents : les Enfants de maître Jacques (ou compagnons du Devoir, ou dévorants), les Enfants de Salomon (ou compagnons du Devoir de Liberté), et les Compagnons du père Soubise (les "gavots " ou les "bons drilles "). Tous revendiquent des origines prestigieuses. Les légendes sur lesquelles elles fondent leurs

17. PoITRINEAU, Abel, Ils travaillaient..., op. cit, p. 68. 
prétentions se rattachent à la construction du temple de Salomon dans l'antique Jérusalem; ces mythes justifient l'union des membres de chaque association mais aussi la forte animosité entre elles.

Ménétra est reçu compagnon du Devoir à Tours : " Je fus reçu compagnon du devoir et les compagnons me firent en entier recopier le rôle ou ce que l'on nomme maître Jacques ou bien le devoir et je fus nommé Parisien le Bienvenu " (p. 50). Il se montre assez discret sur le contenu exact des rites qui jalonnent son ascension; après avoir été initié au devoir, il est fait compagnon fini dans la même ville de Tours, il est nommé premier compagnon à Rochefort, il le sera également à Bordeaux puis à Lyon.

\section{Le voyage}

"Le voyage du $18^{\mathrm{e}}$ est presque toujours ressenti comme une fatalité imposée par la nature [...] On est très anxieux d'en voir la fin [...] C'est que, même dans les meilleures conditions de confort, le voyage n'est jamais une partie de plaisir ${ }^{18}$. " Cette remarque traduit le sentiment immédiat du voyageur devant l'inconfort des conditions d'hébergement et de transport, bien que le XVIII ${ }^{\mathrm{e}}$ siècle connaisse une révolution du transport terrestre ${ }^{19}$. Une hiérarchie est établie entre les axes qui distingue : "les grandes routes", " les routes ", " les grands chemins ", " les chemins royaux" et "les chemins de traverse ". On a remarqué que Ménétra, peu familier des régions qu'il traverse, utilise surtout les grandes routes. En même temps qu'elle s'installe dans le paysage, "la route " s'installe aussi dans les imaginaires. Ménétra aurait parcouru 2500 kilomètres alors que l'on estime à 25000 kilomètres la longueur des routes ouvertes dans le royaume à la veille de la Révolution ${ }^{20}$.

\section{Moyens de locomotion}

Comment "faire route "? Nous pouvons faire la liste des moyens de transport que Ménétra utilise, au premier rang desquels figure, bien entendu, la marche à pied. Mais il monte également à cheval, soit qu'on lui prête une monture, par exemple, près d'Orléans, il rencontre un maquignon qui avait deux chevaux et qui lui en propose un, ou dans un couvent proche de Toulouse : " ce fut un dimanche que je fis mon entrée dans ce couvent suivi du seigneur car nous étions tous deux à cheval et j'allais devant lui parce que les inférieurs vont devant les supérieurs étant la mode du pays " (p. 76). Dans un certain nombre de cas, il monte avec un autre cavalier, par exemple lorsqu'il fait la rencontre de la maréchaussée dans un bois plein de

18. Atlas de la Révolution, fascicule 1, op. cit., p 58.

19. Au départ grâce à la corvée royale, instituée en 1738 par le Contrôleur Général des Finances Orry et la naissance d'une administration spécialisée, les Ponts et Chaussée (Trudaine et Perronet).

20. Sur la question des transports, voir LIVET, Georges, Histoire des routes et des transports en Europe. Des chemins de Saint-Jacques à l'âge d'or des diligences, Strasbourg, Presses universitaires de Strasbourg, 2003, 608-24 p. 
voleurs et de loups : "l'on m'offre de monter en croupe j'accepte » (p. 122). Dans la région de Montélimar, il utilise " la poste aux ânes " (p. 102), terme utilisé pour qualifier la location de ces animaux ${ }^{21}$.

Lorsqu'il voyage a priori plus confortablement, Ménétra écrit qu'il a emprunté " une voiture ". Lors de son second voyage, sur le trajet ParisNîmes : " je fus quinze jours en marche prenant de temps en temps des voitures" (p. 116). Il évoque parfois le fait de demander l'hospitalité aux passagers : il monte, une fois, dans une voiture, après une simple demande : « je lui demandais poliment si elle voulait que je monte dedans elle le voulut bien " (p. 49); une autre fois, il est à souper dans une auberge, il entend une voiture : "Je demandai s'il voulait en payant que je montasse dans sa voiture " (p. 84). Le terme est toujours celui de voiture, sans précision. Pourtant le vocabulaire des historiens est plus varié, chariots ou charrettes, coches et carrosses, qui disposent de huit places, inconfortables et lents, fourgons, ou diligences plus rapides mais plus chères, chaises de poste, encore plus rapides, mais encore plus chères ${ }^{22}$.

Quelques itinéraires spécifiques nécessitent l'utilisation d'un bateau, c'est le cas lors de la navigation qui le mène de Saint-Malo à l'Île d'Yeu mais c'est aussi le cas lorsqu'il emprunte canaux et voies navigables ${ }^{23}$. Il emprunte l'estuaire de la Gironde, de Royan à Bordeaux : " Je partis pour Bordeaux je m'embarquai à Royan nous étions trois nous avions pris des provisions j'étais sur le tillac " (p. 66) et rentre à Paris par l'Yonne : il part d'Auxerre et débarque au port Saint-Paul à Paris, " il [son patron] me conduisit au coche d'eau me paya bien et me donna des provisions pour faire ma route" (p. 108).

\section{Comment voyage-t-on?}

On reconnaît le voyageur à sa tenue, on a bien en tête les images des compagnons itinérants. Chez Ménétra pas de baluchon accroché à la canne, cependant il est souvent question d'un sac; à Toulouse, "comme j'avais laissé un assez bon sac je me débarrassai de ce qui ne m'était pas nécessaire et je partis pour aller à Narbonne " (p. 81); effectivement d'une canne : sur la route de Montpellier, il met dans une voiture où étaient déjà des sacs de grain " [s] on sac avec [s]a canne" (p. 84); et, moins classique, un pistolet : à Béziers, " j'armai mon pistolet car je le portais en route en bandoulière et caché dans les plis de mon habit " (p. 83), plus loin, encore à propos de ce pistolet " pour qu'il ne ballotte point j[e l]'avais attaché avec

21. Agricol Perdiguier évoque aussi cette " poste aux ânes " entre Lunel et Montpellier, Mémoires d'un compagnon, éditions de l'Imprimerie Nationale, 1992, p. 136-137.

22. Voitures publiques, réseau et types de véhicules, Atlas de la Révolution, op. cit., p. $46-47$.

23. Les voies d'eau sont très utilisées, elles sont un moyen de transport populaire, car peu onéreux. Il y a, à la fin de l'Ancien Régime, 8000 km de voies (7000 km de rivières navigables et 1000 de canaux). 
une forte épingle " (p. 84). Dans cet appareil, il se décrit : " je suis ma canne mon tablier en bandoulière sur la route " (p. 119).

Ménétra est beaucoup plus souvent en compagnie que seul pour voyager; le " compagnon de voyage " est l'objet de bien des réflexions. Lorsqu'il les décrit, ses compagnons sont identifiés par leur origine géographique ou par leur métier. Il fait la route de Tours à Angers en compagnie " d'un Rennais " (p. 53), il quitte Poitiers pour Saint-Malo où veulent s'embarquer un compagnon ferblantier angevin et un coutelier langrennois ${ }^{24}$, " entre Carcassonne et Narbonne, [il est] accompagné d'un grand Allemand compagnon culottier " (p. 81), il part de Narbonne " avec un Saint Germain qui avait été capitaine de gavots en Avignon et qui s'était fait recevoir compagnon du Devoir " (p. 82). Il fait souvent route en compagnie de soldats, " je partis [pour Montpellier] avec un sergent du régiment de Béarn " (p. 83), ou de marchands; il part d'une auberge sur la route de Lyon (Nîmes-Lyon) : “ je pars accompagné de plusieurs marchands forains qui se rendaient à Lyon et je fis le voyage avec ces braves gens " (p. 122). Ménétra rencontre parfois des personnages inclassables, mais il indique souvent en quelques mots qu'ils lui inspirent confiance : "Étant sur la grand route un homme assez bien mis qui portait un petit sac sous le bras m'accoste et me demande si je passe à Chalon et qu'il va plus loin je lui dis oui il demande s'il peut avoir ma compagnie" (p. 134).

Entre deux villes, lorsque le voyageur veut faire halte, il fréquente les cabarets ou les auberges, ce qui est l'occasion de comptes avec ses compagnons de route : "nous faisons route comme des amis nous ne brûlons aucun village chacun paie son écot " (p. 134). Ces haltes sont en effet l'occasion de se reposer un instant, il s'arrête à un bourg près d'Orléans " pour se rafraîchir " (p. 41), pour dîner ou souper, pour se distraire (du côté de Toulouse, il entre dans une auberge, où il perd au jeu), pour passer la nuit : " Je fus pour coucher dans une auberge à 4 lieues de Montpellier " (p. 83). Il s'attarde quelquefois lorsque la compagnie lui convient. Ainsi, entre Auch et Toulouse, il passe quatre à cinq jours dans une auberge, où il soigne tout le monde grâce à des remèdes qu'on vient de lui donner. Il fait exceptionnellement halte ailleurs, par exemple dans un château : entre Carpentras et Bédoin : "Nous voilà avec le bourgeois en route il nous fait loger chez un seigneur provençal pour qui il avait travaillé à un joli château. Ce bon seigneur nous fait manger à sa table» (p. 95).

Le texte de Ménétra montre bien que le compagnon voyageur reste le plus souvent en contact avec les siens. Il est souvent question de sommes qu'on lui envoie pour vivre. Ainsi lorsqu'il est à Vendôme : " avec cela que ma mère m'envoyait assez souvent de l'argent faisait que je passais mon temps à merveille " (p. 46). Cette situation ne concerne pas que Ménétra, mais semble une pratique répandue : un compagnon de ses amis « recevait chaque mois au moins 5 à 6 louis " (p. 54). De l'argent lui est envoyé par sa

24. Sans doute langrois (de Langres). 
mère ou par un employeur pour se déplacer. Alors qu'il est à Béziers, on lui envoie de l'argent pour venir à Montpellier; alors qu'il est à Marseille, sa veuve de Nîmes lui envoie 24 francs.

En effet, voyager suppose de disposer d'un peu d'argent. Ménétra raconte avoir fait une fois 40 lieux au moins avec seulement vingt sols. Il couche dans les broussailles, " j'étais presque nu et plein de vermine " (p. 61); il signale cependant, et il semble y attacher une grande importance, qu'il n'a pas mendié. On lui envoie de l'argent par la poste, qu'il utilise également pour les lettres; grâce à laquelle le compagnon voyageur n'est pas coupé de sa famille ${ }^{25}$. C'est aussi par la poste que sa mère lui envoie sa lettre de maîtrise pour le dispenser de la milice. Mais la poste officielle n'est pas la seule solution pour envoyer de ses nouvelles, Ménétra use parfois de la débrouille : à Lyon, " le long de la route j'avais couché en la compagnie d'un homme qui me disait qu'il allait à Paris [...] je luis remis une lettre pour ma grand-mère " (p. 122).

Pour les marchandises, Ménétra utilise les services de la messagerie. Il évoque souvent une cassette, dans laquelle il conserve toutes ses richesses; de fait, la messagerie lui permet de voyager sans s'encombrer, ainsi sa bourgeoise de Nantes lui " envoya [sa] cassette à La Rochelle " (p. 63), il y fait encore allusion du côté d'Auxerre ou de Montereau, " comme je n'avais point de sac que j'avais mis ma cassette à la messagerie " (p. 106). Il la récupère à Paris, avec tous ses habits. Lors de son second départ, puisqu'il pense achever son voyage auprès de sa veuve de Nîmes, il lui envoie sa cassette; lorsqu'il veut quitter Nîmes, il porte encore à la messagerie sa cassette avec ses effets.

\section{Les aléas du voyage}

Si le séjour dans les différentes villes est l'occasion d'enrichissements professionnels et de contacts amicaux, les déplacements entre elles sont l'occasion des anecdotes les plus piquantes qui figurent en bonne place dans les souvenirs de Ménétra.

Ainsi des mauvaises surprises liées aux évènements météorologiques : entre Avignon et Orange, lors d'un orage très fort, la foudre s'abat sur un olivier; ainsi également des malaises physiques surprenant le voyageur sur la route : «lorsque je fus sur la route d'Auxerre comme j'étais en compagnie je me sentis tout à coup une douleur au pied qui devint enflé et ne pouvant aller plus loin je l'[son compagnon de voyage] invitai de passer chez la mère et de m'envoyer une monture pour arriver [...] monté sur un âne ce fut mon entrée à Auxerre " (p. 107).

25. La poste est, au XVIII ${ }^{\mathrm{e}}$ siècle, une entreprise efficace fonctionnant à la satisfaction générale. Le compagnon Toussaint Guillaumou, qui voyage un demi siècle plus tard, évoque ces "secours " des familles envoyés par la poste, Guillaumou, Toussaint, Les confessions d'un compagnon, Jacques Grancher Éditeur, 1996, p. 36. 
Les dangers qui guettent les voyageurs à pied sont logiquement les animaux sauvages, comme les loups : " nous passâmes une forêt où il y avait bien une trentaine de loups qui nous accompagnèrent " (p. 76), ou encore " au milieu d'un bois j'entends des cris de loups j'en vois une légion je tire mon pistolet je fais feu je vois avec plaisir tous prendre la fuite " (p. 120). Plus fréquents, mais pas toujours sympathiques, les chiens censés protéger les paysans qui ne sont pas tendres avec les passants. Aux environs de Niort, Ménétra est mordu par un chien qui déchire ses habits; la réponse de sa maitresse, selon laquelle le chien ne faisait que son devoir, contribue peut-être à la méfiance du compagnon par rapport aux ruraux.

Le voyage est l'occasion de nombreuses rencontres agréables. Ménétra qui s'étend en général beaucoup sur le sujet, évoque certaines rencontres féminines : entre Vendôme et Tours "il m'arriva une charmante aventure ", il monte dans une voiture, et fait le trajet en galante compagnie (p. 49). Pendant sympathique à l'anecdote du chien, sur les routes du Poitou, il est accueilli par un fermier qui lui offre le repas, l'hébergement et le gratifie même de 24 sols le lendemain. Ménétra dit sobrement " il m'arriva un soir une bonne aubaine " (p. 61). Curieux de tout, il apprend, par exemple, l'usage de certains remèdes alors qu'il fait la route avec quelqu'un qui se vante de savoir guérir la fièvre : "il me dit son secret et même me donna de la poudre " (p. 74). Le voyage permet même des rencontres insolites : Ménétra et un compagnon s'occupent d'une femme qui vient d'accoucher sous un olivier, entre Carcassonne et Narbonne.

Les contacts sont également fréquents avec la maréchaussée, des brigades sont établies pour la surveillance routière. Le plus souvent, Ménétra la fuit, mais pas toujours, il est parfois rassuré par sa présence : " au milieu d'un bois [...] j'entends un bruit confus c'est la maréchaussée qui est à la poursuite de voleurs [...] on m'arrête me demande d'où je viens qui suis-je ils me prennent mon arme ma canne et me demandent mes papiers je leur réponds je leur montre ma lettre de maîtrise ils me remettent mes effets me disent que le bois est garni de voleurs et de contrebandiers " (p. 120). À propos des papiers qu'on lui demande, Ménétra ne parle jamais d'autre chose que de sa lettre de maîtrise, il n'évoque jamais un éventuel livret ouvrier.

Parmi les rencontres hostiles, il est souvent arrêté par des compagnons qui cherchent la bagarre; "à trois quarts de lieue de Béziers je pouvais finir mes jours car il y avait huit forts gavots ${ }^{26}$ qui nous attendaient et qui étaient en embuscade" (p. 83); sur la route de Nantes "dans une grande prairie je fis rencontre de deux gavots qui voulaient me prendre mon sac le hasard fit arriver deux compagnons chapeliers qui leur en donnèrent selon leur mérite" (p. 63).

Un certain nombre de récits ont vocation à impressionner le lecteur, ils sont pour cela relativement imprécis et leur date est approximative,

26. Les gavots sont des compagnons " du père Soubise ", association compagnonnique concurrente de celle de Ménétra, les compagnons du devoir. 
c'est le cas d'une rencontre que Ménétra aurait faite de Mandrin, ce qui est impossible, Mandrin étant mort lors du voyage du compagnon ${ }^{27}$. Il écrit qu'il aurait trinqué avec Mandrin : "sur la route de Mâcon j'aperçus l'avantgarde de Mandrin composée de vingt hommes et une demie heure après je l'aperçus avec sa troupe étant à sa tête je le saluai il me le rendit " (p. 108). On sait en revanche que, quelques mois seulement après sa mort, la « littérature mandriniste " était abondante, mêlant vérité historique et légende ${ }^{28}$. Une seconde fois dans son récit, une troupe organisée de gens armés est qualifiée de ce même nom : près de Bourg en Bresse, " dans notre route comme nous étions à manger [...] nous vîmes passer la sœur de Mandrin à cheval accompagnée au moins de 120 hommes armés jusqu'aux dents " (p. 130). Le nom de Mandrin est utilisé presque comme un nom commun, cet épisode confirme la popularité du célèbre contrebandier ${ }^{29}$.

Dans cet ordre d'idées, le récit de Ménétra montre bien que le voyage peut être un contact avec un monde irréel, un monde fantasmé, où circulent des lettres, des objets, des rumeurs. Des informations plus ou moins conformes à la réalité courent à propos des compagnons, sans doute issues des correspondances entre les associations compagnonniques. Lorsqu'il arrive à Toulouse, sa veuve lui dit " qu'il y avait longtemps qu'elle avait entendu parler de [lui] " (p. 75). Mais circulent aussi des rumeurs sur la route et dans les auberges, témoignant de l'attraction populaire pour la culture des faits divers hauts en couleur, des histoires rocambolesques, pour des morts peu banales, du mystère... Près d'Arles, un soir dans une auberge où il arrive avec un camarade, " comme nous arrivâmes un peu tard l'on nous mit dans une chambre où avait décédé un homme " (p. 100); le cadavre est roulé dans un drap et ils lui marchent dessus. Un certain nombre de soirées à l'auberge sont ainsi consacrées à ces récits édifiants : dans une auberge du Dauphiné, l'histoire du fils parti plusieurs années dont le retour a fait mourir sa mère; plus tard " je passai par les Cévennes il commençai à faire nuit lorsque j'arrivai à un village dont j'ai oublié le nom tout était en rumeurs lorsque je fus entré dans une auberge on me dit qu'à celle qui était sur la route, l'homme et la femme avaient assassiné leur enfant " (p. 120). Ces bruits et ces rumeurs se diffusent sur un fond de culture populaire qui entretient le goût du mystère.

L'information circule bien dans le monde des compagnons voyageurs. Lorsque par plaisanterie, Ménétra crée une association de compagnons à laquelle il donne le nom de compagnons de la croûte, il ne faut pas longtemps pour que les compagnons d'autres lieux connaissent l'histoire : "Pendant près de trois mois nous reçûmes des compagnons sans nombre et déjà par toute la France tout voulait être de la croûte " (p. 132).

27. Mandrin est exécuté en 1755, or le départ de Ménétra n'intervient qu'en 1757.

28. GARnot, Benoît, Être brigand du Moyen Âge à nos jours, Paris, Armand Colin, 2013, p. 32 sqq.

29. ANDRIES, Lise (dir.), Cartouche, Mandrin et autres brigands du XVIII siècle, Paris, Desjonquères, 2010, 388 p. 
Les risques sont bien réels pour les voyageurs, parfois victimes de violences. Dès le début de son voyage, Ménétra est capturé et retenu contre son gré : alors qu'il courait après un cheval qu'on lui avait prêté, dans un bois, un homme, qu'il qualifie de " fieffé voleur ", l'oblige à " descendre dans un souterrain [...] près de six semaines j'y suis demeuré " (p. 41). Il fréquente parfois des prisons plus officielles, comme à Poitiers où il est jeté en prison avec les autres compagnons étrangers car il est accusé, à tort, d'avoir dégradé des arbres sur la voie publique. Le voyageur risque souvent d'être détroussé; près de Montpellier, il était monté dans une voiture et s'était endormi, on lui vole son argent, sa culotte, ses souliers.

Le compagnon qui voyage risque de tomber lui-même dans la violence; deux situations sont particulièrement génératrices d'excès, d'une part le contact avec des compagnons d'autres vacations, d'autre part la fréquentation de filles. La rencontre ou la fréquentation de compagnons hostiles génère, sur le tour de France, les "dérouillées " compagnonniques, mais resserre aussi la solidarité des Devoirs, et constitue pour chacun son identité. Les raisons de l'hostilité sont parfois théoriques, elles épousent des rivalités entre associations rivales, mais elles peuvent aussi résulter de contestations dans le travail, de mauvaises plaisanteries d'ateliers, ou d'histoires de femmes. Il arrive même à Ménétra d'être l'auteur d'un viol : ainsi, entre Tours et Angers, lui arrive ce qu'il appelle " une bonne aubaine ". Son camarade et lui rencontrent un jeune garçon et une jeune fille " qui étaient en action ", ils les surprennent, le garçon s'enfuit, le compagnon de Ménétra le suit, "moi je m’amusai avec la fille, moitié bonne volonté le reste de force" (p. 53).

Peut-on tirer un bilan du récit de son Tour de France que fait $a$ posteriori le compagnon vitrier Ménétra? Certes, on voit bien que ce Tour permet un apprentissage professionnel et le récit montre les conditions de voyage d'un artisan, mais on peut tirer du Journal de ma vie quelques remarques supplémentaires.

Ménétra décrit une contre société temporaire de jeunes mâles célibataires, une société bohème, qui refuse ou s'affranchit de tout contrôle, vit sous la tyrannie de la fantaisie, respecte par dessus tout la solidarité du copinage. La culture des compagnons emprunte bien des éléments à la culture populaire (croyance au merveilleux, amour du fait divers, attention prêtée à la rumeur).

Les compagnons sont profondément religieux et, bien qu'il se montre souvent critique à l'égard de l'Église et des ecclésiastiques, Ménétra apparaît bon catholique, comme le montre cette discussion qui l'oppose à des protestants : " Je leur dis qu'il y avait d'honnêtes hommes dans l'une comme dans l'autre maison Mais un me dit vous croyez à votre saint Jérôme et saint Augustin ce sont deux jean foutres moi sans savoir bien ce que je disais je lui dis qu'il ne sait ce qu'il dit Après avoir bien argumenté il finit par me dire 
que ceux qui changent de religion sont des jean foutres Je lui réponds que Luther et Calvin en ont changé... il se jette sur moi et plusieurs de ces forcenés veulent me tuer " (p. 120). Il est encore sauvé par la maréchaussée... Les moqueries par rapport à la dévotion se transforment parfois en hymne à la tolérance, il se moque de la maîtresse qui, à Vendôme, considérait qu'il avait fait un pacte avec le diable; il travaille dans la demeure de campagne de l'évêque d'Agen et se rappelle : "C'est dans ce pays où je vis tous ces paysans fanatisés qui apportaient à cet évêque tous les jours des perdrix et des faisans " (p. 73); il voit avec consternation le fanatisme outré de la Sologne, et ses rencontres avec des protestants et des juifs lui donnent l'occasion de relativiser.

Mais par-dessus tout, Ménétra reste toujours un urbain satisfait de son urbanité, et fier de son caractère parisien, sans cesse évoqué dans le journal. Deux exemples parmi bien d'autres : dans l'épisode de Poitiers où il est jeté en prison ainsi que d'autres compagnons étrangers, "les juges en voulaient plus particulièrement à moi parce que disaient-ils j'étais de Paris et avais l'air plus avisé et que je leur répondais avec plus d'adresse que les autres " (p. 59); à propos de son voyage en bateau Royan-Bordeaux, il écrit : " des passagers comme il faut me demandèrent de quel pays j'étais lorsque je leur eus dit que j'étais de Paris, ils ne voulurent plus que je les quitte et m'invitèrent à faire bombance avec eux " (p. 66). Son tour de France est pour le compagnon un réservoir de souvenirs, bons ou mauvais moments, mais le voyage confirme le Parisien dans la conscience de sa supériorité.

\section{RÉSUMÉ}

Quoique le Tour de France soit voyage et que cette expérience façonne l'identité des compagnons itinérants, et bien que le journal de Ménétra nous livre des informations sur l'organisation des associations compagnonniques dans différentes villes, cette communication s'intéressera aux faits, gestes et pensées de Ménétra lors de ses déplacements de ville en ville. Elle évoquera successivement l'itinéraire du compagnon, les moyens de transport qu'il utilise, ses compagnons de voyage et d'une manière générale le monde de la route.

\section{ABSTRACT}

Though the tour of France was a journey and that this experience formed the identity of itinerant companions, and though Ménétra's journal offers information on the organisation of companionships in different towns, this study will focus on the actions and thoughts of Ménétra as he moved from town to town. It will describe the route followed by the companion, the type of transport used, who his fellow travellers were and, more generally, the world in which such journeys were undertaken. 